\title{
Diagnosis of vascular rejection of a transplanted kidney- a case report
}

Authors: Katarzyna Drelich ${ }^{1}$, Olga Pustelniak ${ }^{2}$, Karol Bochyński ${ }^{1}$, Maryla Kuczyńska ${ }^{3}$, Magdalena Maria Woźniak ${ }^{4}$

1 Students' Scientific Society at the Department of Pediatric Radiology, Medical University of Lublin, Lublin, Poland

2 Students' Scientific Society at the Department of Interventional Radiology and Neuroradiology, Medical University of Lublin, Lublin, Poland

${ }^{3}$ Department of Interventional Radiology and Neuroradiology, Medical University of Lublin, Lublin, Poland

${ }^{4}$ Department of Pediatric Radiology, Medical University of Lublin, Lublin, Poland

DOI: https://doi.org/10.26800/LV-142-supp5-21

\section{Background:}

Kidney transplantation is the method of choice for the treatment of renal failure. After such surgery some complications may occur including rejection of the new organ. The vascular rejection may be acute and is associated with a reduction in the lumen of the artery due to the presence of inflammatory exudate and chronic due to the dynamic proliferation of fibrous connective tissue in the inner membrane of the vessel. The aim of study was to evaluate the effectiveness of ultrasound in the diagnosis of vascular rejection of a transplanted kidney.

\section{Case presentation:}

Our patient is a 19-year-old woman after kidney transplantation due to glomerulonephritis. Up to 6 months after the transplant, the results of laboratory tests and imaging examinations (ultrasound) were normal. Moreover the patient was urinating in the correct amount. After 6 months, the patient had an increasing decrease in the 24-hour urine collection. In subsequent control examinations, despite modifying the immunosuppressive treatment, an increase in abnormal values of laboratory parameters such as creatinine, uric acid and urea were found. Moreover, there were found increasing abnormalities in the morphological ultrasound image of the kidney and a pathological increase in the values of vascular resistance indexes (PI and $\mathrm{RI})$. In the next control examination, massive renal edema was found with a complete lack of corticospinal differentiation and the presence of only systolic peaks in arterial branches. Based on the results of the tests and the clinical condition, the patient was qualified for removal of the transplanted kidney and treatment with dialysis.

\section{Conclusions:}

Doppler Ultrasound examination is an effective imaging method in the diagnosis and monitoring of vascular rejection of a transplanted kidney

Keywords: kidney, transplantation, US Doppler 